Wagner, Christian; Arora, Richa: Indiens Ringen um die Staatsbürgerschaft: die Modi-Regierung forciert ihre nationalistische Agenda / Christian Wagner, Richa Arora. - Berlin: SWP, [Januar 2020] - [Elektronische Ressource (4S.)] (SWP-Aktuell; 2)

Wong, Mathew Y. H.; Kwong, Ying-ho: One formula, different trajectories: China's coalition-building and elite dynamics in Hong Kong and Macau / Mathew Y. H. Wong, Ying-ho Kwong. - In: Critical Asian studies (Abingdon), 52 (2020) 1, S. 44-66

Yamamoto, Keizō: Regelungen der irreführenden Internetwerbung im japanischen Verbraucherrecht / Keizō Yamamoto. - In: Zeitschrift für japanisches Recht (Köln), 24 (2019) 48, S. 17-47

Zhu, Lijiang: Treaties in the Chinese legal order: discourses, developments and debates / Zhu Lijiang. - In: China: CIJ (Singapore), 17 (2019) 4, S. 135-154

\title{
LATEINAMERIKA / LATIN AMERICA
}

Alvarado Rodríguez, María Eugenia: Modernidad y derecho en América Latina: acumulación capitalista, desarrollo, naturaleza y movimientos sociales contrahegemónicos / María Eugenia Alvarado Rodríguez. - México, D.F: Universidad Nacional Autónoma de México, Centro de Investigaciones Interdisciplinarias en Ciencias y Humanidades, 2018 - 398S., Lit.Hinw. (Colección Debate y reflexión) ISBN 978-607-30-1050-4; 978-607-02-9726-7

Assessing the left turn in Ecuador / Francisco Sánchez, Simón Pachano (eds.). - Cham: Springer International Publishing, 2020 - xxx, 373S., graph. Darst. ISBN 978-3-030-27624-9

Bolivia (plurinational state of)'s constitution of 2009 / Estado Plurinacional de Bolivia. - Oxford: Oxford University Press, 2019 - [Elektronische Ressource (131S.)]

Bachelet, Michelle: De la ONU a un régimen que no rinde cuentas y niega las violaciones de derechos humanos que ha cometido / Michelle Bachelet. - In: Envio (Managua), 38 (2019) 451, S. 14-25

de la Cruz Prego, Fernando: Una década de construcción del estado desarrollista post-neoliberal en Bolivia: luces, sombras y faros / Fernando de la Cruz Prego. - Madrid: Fundación Carolina, 2019 [Elektronische Ressource (35S., graph. Darst.)] (Documento de trabajo; 18)

Ecuador 2018 Human Rights Report / USA. Bureau of Democracy, Human Rights, and Labor. USA: United States Department of State, Bureau of Democracy, Human Rights and Labor, 2019 [Elektronische Ressource (32S.)]

Esquirol, Jorge L.: Ruling the law: legitimacy and failure in Latin American legal systems / Jorge L. Esquirol. - Cambridge: Cambridge University Press, 2019 - 302S. ISBN 978-1-107-17839-7 (ASCL studies in comparative law)

Freeman, Mark; Orozco Abad, Iván: Negotiating transitional justice: firsthand lessons from Colombia and beyond / Mark Freeman, Iván Orozco. - Cambridge: Cambridge University Press, 2020 ix, 255S. ISBN 978-1-107-18756-6; 978-1-316-63815-6

Fuentes Julio, Claudia; Ibrahim, Raslan: A human rights approach to conflict resolution / Claudia Fuentes-Julio, Raslan Ibrahim. - In: Ethics \& international affairs (Cambridge), 33 (2019) 3, S. $271-273$ 
Houle, Christian; Miller, Michael K.: Social mobility and democratic attitudes: evidence from Latin America and Sub-Saharan Africa / Christian Houle, Michael K. Miller. - In: Comparative political studies (Thousand Oaks, Calif.), 52 (2019) 11, S. 1610-1647

Kunath, Wolfgang: Bolsonaro Politik: irrational und wissenschaftsfeindlich / Wolfgang Kunath. - In: Brasilien-Nachrichten (Freiburg i. Br), (2019) 160, S. 4-7

Madrid, Raúl L.: The partisan path to democracy: Argentina in comparative perspective / Raúl L. Madrid. - In: Comparative political studies (Thousand Oaks, Calif.), 52 (2019) 10, S. 1535-1569

Martínez Espinosa, Luisa Fernanda; Peláez Lozada, Karen Vanessa: El estado actual del cumplimiento de las medidas de satisfacción ordenadas por la corte interamericana de derechos humanos, en las sentencias condenatorias contra el estado colombiano / Luisa Fernanda Martínez Espinosa, Karen Vanessa Peláez Lozada. - In: Revista latinoamericana de derechos humanos (Heredia), 30 (2019) 1, S. 227-252

Monsalve Egaña, Sebastián: Crisis política en Chile: del estallido social al conflicto político / Sebastián Monsalve Egaña. - Santiago de Chile: UAM Red Econolatin, 2019 - [Elektronische Ressource (11S.)] (Nota prospectiva de Econolatin/UAM Red Econolatin; 5)

Moreira, Constanza: Tiempos de democracia plebeya: presente y futuro del progresismo en Uruguay y América Latina / Constanza Moreira. - Ciudad Autónoma de Buenos Aires: CLASCO, 2019 424S., graph. Darst. ISBN 978-987-722-444-3

Querós Vargas, Emilia: Derechos humanos y realidad educativa / Emilia Quirós Vargas. - In: Revista latinoamericana de derechos humanos (Heredia), 30 (2019) 1, S. 47-60

Ramos Quiroz, Francisco: La defensa de los derechos humanos en sede universitaria en México: avances en la materia a 32 años de su establecimiento / Francisco Ramos Quiroz. - In: Revista latinoamericana de derechos humanos (Heredia), 30 (2019) 1, S. 169-192

Roht-Arriaza, Naomi; Martínez, Santiago: Venezuela, Grand Corruption, and the International Criminal Court / Naomi Roht-Arriaza, Santiago Martínez. - San Francisco: University of California, UC Hastings Law, 2019 - [Elektronische Ressource (35S.)] (Research Paper; 340)

Routledge handbook of law and society in Latin America: Handbook of law and society in Latin America / Rachel Sieder, Karina Ansolabehere, Tatiana Alfonso (eds.). - New York: Routledge, Taylor \& Francis Group, 2019 - xviii, 475S., Ill. (Routledge handbooks) ISBN 978-1-315-64519-3; 978-1-317-29127-5; 978-1-317-29128-2; 978-1-317-29126-8

Zamudio-González, Laura: The International Commission against Impunity in Guatemala (CICIG): a self-directed organization / Laura Zamudio-González. - In: Global governance (Boulder), 25 (2019) 3, S. 418-444

Zilla, Claudia; Keseberg, David: Venezuela: Menschenrechte im Ausnahmezustand / Claudia Zilla, David Keseberg. - In: Zeitschrift für Menschenrechte (Schwalbach, Ts), 13 (2019) 2, S. 38-56

\section{NAHER UND MITTLERER OSTEN / NEAR AND MIDDLE EAST}

Abbott, Pamela; Teti, Andrea; Sapsford, Roger: The tide that failed to rise: young people's politics and social values in and after the Arab uprisings / Pamela Abbott, Andrea Teti, Roger Sapsford. In: Mediterranean politics (Abingdon), 25 (2020) 1, S. 1-25 